\title{
Edge lengths determining tetrahedrons
}

\author{
Karl Wirth and André S. Dreiding \\ Karl Wirth received his Ph.D. in mathematics from the ETH Zürich. He then became a \\ mathematics teacher in Zürich. Since his retirement he persues his interests in mathe- \\ matical chemistry. \\ André S. Dreiding is professor emeritus for organic chemistry at the University of \\ Zürich. More than fifty years ago he constructed molecular models the widespread \\ applications of which stimulated the cooperation with mathematicians.
}

\section{Introduction}

For a tetrahedron to actually exist, the edge lengths of each of its four faces evidently must obey the triangle inequality. This condition is necessary but not sufficent for six edge lengths to make up a tetrahedron. There does, for example, not exist a tetrahedron with five edges of length 4 and one edge of length 7 , even though the triangle inequalities are fulfilled. Just consider two equilaterals with edge length 4 as faces of a tetrahedron; the remaining edge length must be smaller than $4 \sqrt{3}(<6.93)$, since this is the extreme value reached when the tetrahedron becomes degenerate (see Fig. 1).

When are six given lengths the edge lengths of some tetrahedron? This question has been addressed to already several times in the literature (Menger, Blumenthal, Dekster and Wilker, Herzog, see below), mostly even for the general case of $d$-dimensional simplices. The present work, as an offshoot of our original investigations concerning tetrahedral structures in organic chemistry, is restricted to the 3-dimensional case. This restriction

Für die Seitenlängen jedes Dreiecks gilt die Dreiecksungleichung. In Analogie dazu stellen die Autoren die Frage, welche Beziehung zwischen den Kantenlängen eines beliebigen Tetraeders bestehen. Unter Verwendung von Cayley-Menger Determinanten beweisen sie im Hauptsatz dieser Arbeit mit elementaren Mitteln notwendige und hinreichende Bedingungen dafür, dass sechs positive reelle Zahlen Kantenlängen eines Tetraeders sein können. Mit Hilfe dieses Hauptsatzes werden dann weitere Probleme betreffend Festlegung von Tetraedern durch Kantenlängen gelöst. Dabei geht es auch um die Anzahl anisometrischer Tetraeder, die mit bestimmten Kantenlängen möglich sind. Die in diesem Beitrag behandelten Probleme haben ihren Ursprung in der Untersuchung von Tetraeder-Strukturen in der Chemie. 


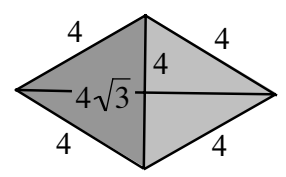

Fig. 1

enables us to offer a well-rounded and readily accessible approach to the above question. We provide theorems with elementary proofs, based on a single, essentially geometrical condition necessary and sufficient for the existence of a tetrahedron (Lemma 2.1).

\section{Preliminaries}

As the concept of triangle inequality plays an important role in this paper we first outline our pertinent terminology: If $(a, b, c)$ is a triple of positive real numbers, the system of inequalities $a<b+c \wedge b<a+c \wedge c<a+b$ is called a triangle inequality (note the singular). We say that $(a, b, c)$ obeys or fulfills the triangle inequality if all three inequalities hold. It is a property of an unfulfilled triangle inequality, that only one of the three inequalities does not hold: Indeed, let there be, for instance, $b+c \leq a$, then we have equivalently $2 b+2 c \leq a+b+c$ which implies $b<a+c$ and $c<a+b$. In the following, we distinguish between a triangle inequality applied to lengths, for short L-inequality, and a triangle inequality applied to angles, more precisely their measures (here in degrees), for short A-inequality.

To start our subject we give some definitions: Let $S=(x, y, z, \bar{x}, \bar{y}, \bar{z})$ be a sextuple of positive real numbers called the lengths of $S$. If there exists a (nondegenerate) tetrahedron $T$ such that the lengths of $S$ are the edge lengths of $T$, as shown in Fig. 2, we say that $S$ is tetrahedral or that $S$ determines $T$. The order of the lengths in a tetrahedral sextuple $S$ indicates in which way the edges of $T$ are arranged: The first three lengths $x, y$ and $z$ of $S$ become the lengths of edges joining in a vertex of $T$ and the last three lengths of $S$, denoted by the same letters but overlined $\bar{x}, \bar{y}$ and $\bar{z}$, become the lengths of the corresponding opposed edges of $T$. Clearly, all tetrahedrons determined by the same sextuple $S$ are isometric, and a given tetrahedron $T$ can be determined in 4 ! ways by sextuples (they are mutually different if $T$ is asymmetric).

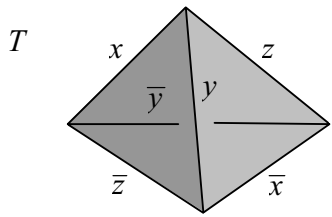

Fig. 2

Given a sextuple $S=(x, y, z, \bar{x}, \bar{y}, \bar{z})$, each of the triples $(x, y, \bar{z}),(x, \bar{y}, z),(\bar{x}, y, z)$ and $(\bar{x}, \bar{y}, \bar{z})$ is called a face-triple of $S$. If all four face-triples obey the L-inequality we refer to $S$ as a facial sextuple. The four triangles thus determined are called faces 
of $S$. A tetrahedral sextuple $S$ is facial but the reverse is not true, as the counter-example $S=(4,4,4,4,4,7)$ illustrates (see Section 1$)$.

When the four faces of a facial (but not necessarily tetrahedral) sextuple $S$ are arranged in a plane, as shown in Fig. 3, we speak of the net of $S$. In Fig. 3, any three angles marked by the same shade of gray make up what we call a vertex-triple of $S$ (inside a triple choose any order). Note that the sum of the angles of one vertex-triple can be $\geq 360^{\circ}$ so that two faces of the net will overlap (except where this triple consists of three outside angles which are shown in Fig. 3 by the darkest shade of gray).

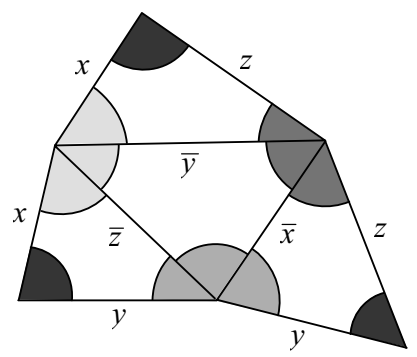

Fig. 3

We can now reformulate the question posed in Section 1, more precisely, as follows: Which facial sextuples $S$ are tetrahedral or, in other words, which condition must be satisfied for the net of a facial sextuple $S$ to be the net of a tetrahedron $T$ ? Obviously, $S$ is tetrahedral if a tetrahedron $T$ can be generated by inward folding of the three outside faces of the net of $S$. From the triangle inequality for spherical triangles it follows that this is possible exactly if one of the vertex-triples of $S$ with sum $<360^{\circ}$ obeys the A-inequality (in that case, of course, this also holds for the other three vertex-triples). By referring to a vertex-triple containing only acute angles as an acute vertex-triple, it is possible to state a sharpened version of this tetrahedrality condition as follows:

Lemma 2.1. A facial sextuple $S$ is tetrahedral exactly if it has an acute vertex-triple obeying the A-inequality.

Proof. It suffices to show that a tetrahedral sextuple $S$ has at least one acute vertex-triple (in other words, that any tetrahedron has at least one vertex where the three adjacent face angles are acute): Assume that the four vertex-triples of $S$ would not be acute, i.e., they all contain an angle $\geq 90^{\circ}$. Since the tetrahedrality of $S$ demands that all vertex-triples obey the A-inequality, the sum of the angles of each vertex-triple is $>180^{\circ}$ and thus the total sum of all twelve face angles becomes $>720^{\circ}$, a contradiction because this sum must be $4 \cdot 180^{\circ}=720^{\circ}$. 


\section{Main theorem}

The following main Theorem 3.1 was originally proved by Menger [8], not only for tetrahedrons but for the general case of $d$-dimensional simplices. For tetrahedrons, we give another derivation based on Lemma 2.1. A similar reasoning, albeit in the opposite direction, can be found in an early paper of Blumenthal [1].

Theorem 3.1. A sextuple $S=(x, y, z, \bar{x}, \bar{y}, \bar{z})$ is tetrahedral exactly if (i) $S$ is facial and (ii) $\mathrm{D}(S)$ is positive, where $\mathrm{D}(S)$ denotes the so-called Cayley-Menger determinant ${ }^{1}$ :

$$
\mathrm{D}(S)=\left|\begin{array}{ccccc}
0 & x^{2} & y^{2} & z^{2} & 1 \\
x^{2} & 0 & \bar{z}^{2} & \bar{y}^{2} & 1 \\
y^{2} & \bar{z}^{2} & 0 & \bar{x}^{2} & 1 \\
z^{2} & \bar{y}^{2} & \bar{x}^{2} & 0 & 1 \\
1 & 1 & 1 & 1 & 0
\end{array}\right| .
$$

Proof. As already pointed out the condition (i) is necessary, so that Lemma 2.1 can be applied: Without loss of generality, the following angles $\alpha, \beta$ and $\gamma$, given by the cosine law, can be chosen as those of an acute vertex-triple of $S$ :

$$
\cos \alpha=\frac{y^{2}+z^{2}-\bar{x}^{2}}{2 y z}, \quad \cos \beta=\frac{x^{2}+z^{2}-\bar{y}^{2}}{2 x z}, \quad \cos \gamma=\frac{x^{2}+y^{2}-\bar{z}^{2}}{2 x y} .
$$

Obeying the A-inequality means that

$$
\alpha<\beta+\gamma \wedge \beta<\alpha+\gamma \wedge \gamma<\alpha+\beta \Leftrightarrow|\beta-\gamma|<\alpha<\beta+\gamma .
$$

Since $0 \leq|\beta-\gamma|$ and $\beta+\gamma<180^{\circ}$ and since the cosine decreases monotonically in the interval $\left[0^{\circ}, 180^{\circ}\right]$, one can equivalently write

$$
\cos |\beta-\gamma|>\cos \alpha>\cos (\beta+\gamma),
$$

respectively

$$
\cos (\beta-\gamma)>\cos \alpha>\cos (\beta+\gamma) .
$$

Using trigonometric relations and other transformations, the following further equivalent inequalities are obtained:

$$
\begin{aligned}
& \cos \beta \cos \gamma+\sin \beta \sin \gamma>\cos \alpha>\cos \beta \cos \gamma-\sin \beta \sin \gamma \\
\Leftrightarrow & \sin \beta \sin \gamma>\cos \alpha-\cos \beta \cos \gamma>-\sin \beta \sin \gamma \\
\Leftrightarrow & \sin ^{2} \beta \sin ^{2} \gamma>(\cos \alpha-\cos \beta \cos \gamma)^{2} \\
\Leftrightarrow & \left(1-\cos ^{2} \beta\right)\left(1-\cos ^{2} \gamma\right)>\cos ^{2} \alpha-2 \cos \alpha \cos \beta \cos \gamma+\cos ^{2} \beta \cos ^{2} \gamma \\
\Leftrightarrow & 1+2 \cos \alpha \cos \beta \cos \gamma-\cos ^{2} \alpha-\cos ^{2} \beta-\cos ^{2} \gamma>0 .
\end{aligned}
$$

\footnotetext{
${ }^{1}$ The determinant was given this name (1953) by Leonhard M. Blumenthal in [3, p. 99] with the justification that it had been proposed initially (1841) by the young Arthur Cayley in his very first paper dealing with certain point arrangements [5, pp. 267-271] and had been subsequently used more than eigthy years later (1923) by Karl Menger to solve imbedding problems in Euclidean space [8, pp. 120 and 133].
} 
If the cosines on the left side of this inequality are expressed by the terms of (3.2) one arrives, by some calculation, at

$$
\frac{\mathrm{D}(S)}{8 x^{2} y^{2} z^{2}}>0 .
$$

Since the denominator is positive it follows that the facial sextuple $S$ is tetrahedral exactly if condition (ii) holds.

\section{Remarks}

(1) Both conditions of Theorem 3.1 must be verified in order to ensure that a sextuple $S$ is tetrahedral. In general, (i) does not imply (ii): Take (again, see Section 1) the facial, but not tetrahedral $S=(4,4,4,4,4,7)$ where $\mathrm{D}(S)=-1568$. Conversely, (ii) does not necessarily imply (i): Just consider $S=(1,1,3,5,1,3)$ where $\mathrm{D}(S)=$ 468 but $S$ is not facial.

(2) If $S$ is a tetrahedral sextuple the Cayley-Menger determinant $\mathrm{D}(S)$ has an immediate geometrical significance: $\mathrm{D}(S)=288 V^{2}$, where $V$ denotes the volume of a tetrahedron determined by $S$. This formula, the 3-dimensional analogue of Heron's formula, has already been proved by the painter Piero della Francesca ( 1412-1492), (see [9]); the reader may verify it by using, for instance, the scalar triple product and vector algebra. Note that a tetrahedron will be degenerate exactly if $S$ is a facial sextuple and if $\mathrm{D}(S)=0$. In other words, $\mathrm{D}(S)=0$ of a facial $S$ expresses the relationship among the distances between four points in a plane. Indeed, for the facial $S=(4,4,4,4,4,4 \sqrt{3})$ which determines the degenerate tetrahedron shown in Fig. 1, for example, one obtains $\mathrm{D}(S)=0$.

(3) The Cayley-Menger determinant $\mathrm{D}(S)$ is a polynomial of degree 6 in 6 variables. The following specific representation of $\mathrm{D}(S)$, where $S=(x, y, z, \bar{x}, \bar{y}, \bar{z})$, shows its symmetry properties:

$$
\begin{aligned}
\mathrm{D}(S)= & 2 x^{2} \bar{x}^{2}\left(y^{2}+\bar{y}^{2}+z^{2}+\bar{z}^{2}-x^{2}-\bar{x}^{2}\right) \\
& +2 y^{2} \bar{y}^{2}\left(z^{2}+\bar{z}^{2}+x^{2}+\bar{x}^{2}-y^{2}-\bar{y}^{2}\right) \\
& +2 z^{2} \bar{z}^{2}\left(x^{2}+\bar{x}^{2}+y^{2}+\bar{y}^{2}-z^{2}-\bar{z}^{2}\right) \\
& +\left(x^{2}-\bar{x}^{2}\right)\left(y^{2}-\bar{y}^{2}\right)\left(z^{2}-\bar{z}^{2}\right)-\left(x^{2}+\bar{x}^{2}\right)\left(y^{2}+\bar{y}^{2}\right)\left(z^{2}+\bar{z}^{2}\right) .
\end{aligned}
$$

$\mathrm{D}(S)$ is invariant under 4 ! permutations of the six variables. These permutations correspond to the 4 ! ways a tetrahedron can be determined by (tetrahedral) sextuples.

(4) The condition (i) can also be expressed by Cayley-Menger determinants as follows: $S$ is facial exactly if $\mathrm{D}_{j}(S)$ is negative for all $j$ with $1 \leq j \leq 4$, where $\mathrm{D}_{j}(S)$ is the $(j, j)$-minor of $\mathrm{D}(S)$, i.e., the determinant of the submatrix obtained from the matrix involved in (3.1) by deleting the $j$-th row and the $j$-th column. Consider $j=1$ (the same reasoning can be used for $j \neq 1$ ):

$\mathrm{D}_{1}(S)=\left|\begin{array}{cccc}0 & \bar{z}^{2} & \bar{y}^{2} & 1 \\ \bar{z}^{2} & 0 & \bar{x}^{2} & 1 \\ \bar{y}^{2} & \bar{x}^{2} & 0 & 1 \\ 1 & 1 & 1 & 0\end{array}\right|=-(\bar{x}+\bar{y}+\bar{z})(\bar{x}+\bar{y}-\bar{z})(\bar{y}+\bar{z}-\bar{x})(\bar{z}+\bar{x}-\bar{y})$, 
which is negative exactly if the face-triple $(\bar{x}, \bar{y}, \bar{z})$ obeys the L-inequality. Furthermore, if $\mathrm{D}_{1}(S)$ is negative, Heron's formula for the area $V_{1}$ of the face determined by $(\bar{x}, \bar{y}, \bar{z})$ is obtained: $V_{1}=\frac{1}{4} \sqrt{-\mathrm{D}_{1}(S)}$.

(5) If $\mathrm{D}(S)$ is positive it suffices to check that one of the four determinants $\mathrm{D}_{j}(S)$ is negative (i.e. that just one of the four face-triples obeys the L-inequality) for a sextuple $S$ to be tetrahedral (see remarks in [2]). Let, for instance, $\mathrm{D}_{1}(S)$ be negative: Since $\mathrm{D}_{1}(S) \cdot \mathrm{D}_{j}(S)=2 t^{2} \mathrm{D}(S)+\left(\mathrm{D}_{1 j}(S)\right)^{2}$, where $\mathrm{D}_{1 j}(S)$ is the $(1, j)$-minor of $\mathrm{D}(S)$ and where $t=\bar{x}$ for $j=2, t=\bar{y}$ for $j=3$ and $t=\bar{z}$ for $j=4$, the determinants $\mathrm{D}_{j}(S)$ with $2 \leq j \leq 4$ are negative, too. Theorem 3.1 can now be sharpened; it then corresponds to Blumenthal's formulation for $d$-dimensional simplices in [3] when $d=3$ :

$S$ is tetrahedral $\Leftrightarrow \mathrm{D}(S)$ is positive and $\mathrm{D}_{1}(S)$ is negative.

\section{Acute-facial sextuples and completely tetrahedral sets}

Given a triple $(a, b, c)$ of positive real numbers, the triangle inequality for their squares, i.e., $a^{2}<b^{2}+c^{2} \wedge b^{2}<a^{2}+c^{2} \wedge c^{2}<a^{2}+b^{2}$, is said to be a $Q$-inequality. A fulfilled Q-inequality implies a fulfilled L-inequality: Indeed, from $a^{2}<b^{2}+c^{2}$, for instance, it follows $a^{2}<(b+c)^{2}$ which is equivalent to $a<b+c$.

We speak of an acute-facial sextuple $S$ if all of its four face-triples obey the Q-inequality. Since a fulfilled Q-inequality implies a fulfilled L-inequality it follows that an acute-facial $S$ is facial. In addition, the cosine law implies that $S$ is acute-facial exactly if all vertextriples (i.e. all twelve face angles) are acute. Furthermore, we have:

Theorem 4.1. An acute-facial sextuple $S$ is tetrahedral.

Proof. Assume that an acute-facial $S$ would not be tetrahedral. Then, according to Lemma 2.1 , the four acute vertex-triples of $S$ do not obey the A-inequality. This implies that the sum of the angles of each vertex-triple is $<180^{\circ}$ and thus the total sum of all twelve face angles becomes $<720^{\circ}$, again a contradiction to the required $720^{\circ}$.

Up to now we considered given sextuples and asked whether they are tetrahedral or not. We shall now ask from which supply the lengths of sextuples can be freely chosen so as to guarantee tetrahedrality. A (non-empty) set $W$ of positive real numbers, in the present context called lengths, is said to be a completely tetrahedral set if all sextuples $S$ with lengths from $W$ are tetrahedral. The following Theorem 4.2, in essence, is a consequence of Theorem 4.1. Another derivation of almost the same result and an extension to $d$ dimensional simplices have been given by Dekster and Wilker in [6].

Theorem 4.2. A set $W$ of lengths is completely tetrahedral exactly if it is a subset of an interval $I=[t, t \sqrt{2}]$ containing at most one endpoint of $I$.

Proof. Of course, $t$ must be positive. On the one hand, assume that $W$ is a subset of an interval $I=[t, t \sqrt{2}]$, with $W$ not containing both $t$ and $t \sqrt{2}$. From $(t \sqrt{2})^{2}=t^{2}+t^{2}$ it follows that, for any $a, b$ and $c$ in $W$, one has $a^{2}<b^{2}+c^{2}$ so that all triples with lengths 
from $W$ obey the Q-inequality. Hence, all sextuples $S$ with lengths from $W$ are acutefacial and, by Theorem 4.1, tetrahedral, meaning that $W$ is completely tetrahedral. On the other hand, let $W$ be a set containing lengths $a$ and $b$ with $a \sqrt{2} \leq b$. Then the CayleyMenger determinant of the sextuple $\tilde{S}=(a, a, b, a, a, b)$ is $\mathrm{D}(\tilde{S})=4 b^{4}\left(2 a^{2}-b^{2}\right) \leq 0$. Thus, according to Theorem $3.1, \tilde{S}$ is not tetrahedral, meaning that $W$ is not completely tetrahedral.

\section{Remarks}

(1) Note that $\mathrm{D}(\tilde{S})=0$ for $b=a \sqrt{2}$, so that the facial $\tilde{S}$ determines a degenerate tetrahedron; its edges become the sides and diagonals of a square.

(2) All sextuples $S$ with lengths obtained from a completely tetrahedral set $W$ are acutefacial. The converse evidently is not true; just consider $S=(2,2,2,1,1,1)$ as a counter-example.

Which completely tetrahedral set $U$ consists of the smallest six successive integers? Since $n=13$ is the smallest solution of the diophantine inequality $n+5<n \sqrt{2}$ it follows from Theorem 4.2:

$$
U=\{13,14,15,16,17,18\}
$$

\section{Properties of tetrahedral spawning sextuples}

A sextuple $S_{\mathrm{sp}}=(a, e, c, b, d, f)$ with $a \geq b \geq c \geq d \geq e \geq f$ will be called a spawning sextuple. A remarkable property of spawning sextuples has been detected by Herzog [7]:

Theorem 5.1. If a spawning sextuple $S_{\mathrm{sp}}$ is tetrahedral all sextuples $S$ obtained by permuting the lengths of $S_{\mathrm{sp}}$ are tetrahedral, too.

Outline of a proof. Let $S_{\mathrm{sp}}$ be a tetrahedral spawning sextuple. We use Theorem 3.1: Since $(a, e, f)$ is a face-triple of $S_{\mathrm{sp}}$ one has $a<e+f$, where $a$ is the largest and $e$ and $f$ are the two smallest lengths of $S_{\mathrm{sp}}$, so that all triples with lengths from $S_{\mathrm{sp}}$ obey the L-inequality. Thus, all sextuples $S$ obtained by permuting the lengths of $S_{\text {sp }}$ satisfy (i). It remains to verify (ii) for all these $S$. From here on we follow the ideas of the proof in [7]: In a first step, it is shown by calculation that the minimum of all $\mathrm{D}(S)$ is reached by $\mathrm{D}\left(S_{\mathrm{sp}}\right)$, $\mathrm{D}\left(S_{1}\right)$ or $\mathrm{D}\left(S_{2}\right)$, where $S_{1}=(a, e, d, b, f, c)$ and $S_{2}=(a, e, d, b, c, f)$. In a second step, primarily geometrical considerations are used to prove that if $\mathrm{D}\left(S_{\mathrm{sp}}\right)$ is positive then both $\mathrm{D}\left(S_{1}\right)$ and $\mathrm{D}\left(S_{2}\right)$ are positive, too. Details of these two steps can be found in [7] for the case $a>b>c>d>e>f$, but both steps can also be applied to the general case $a \geq b \geq c \geq d \geq e \geq f$.

\section{Remarks}

(1) Calling a tetrahedron determined by a spawning sextuple $S_{\mathrm{sp}}=(a, e, c, b, d, f)$ a spawning tetrahedron (see Fig. 4), Theorem 5.1 can be stated as follows: All conceivable anisometric tetrahedrons obtained by rearranging the edges of the spawning tetrahedron exist; including the latter there are maximally 30 of them, as will be explained in Section 6. 


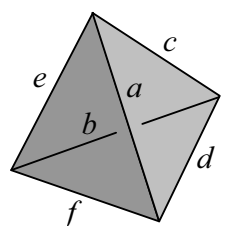

Fig. 4

(2) A tetrahedron with pairwise different edge lengths is named here a scalene tetrahedron. Note that, among all conceivable anisometric scalene tetrahedrons with edge lengths 120,110,100, 90, 80 and 61 (here exactly 30), only the spawning tetrahedron does not exist (see remark in [7]). By the way, there does not exist a scalene tetrahedron with edge lengths 194, 193, 100, 99, 98 and 97 even though all triples taken from these lengths obey the L-inequality (see remark in [4]).

(3) In the case of a spawning sextuple $S_{\mathrm{sp}}=(a, e, c, b, d, f)$ one finds that (ii) implies (i) (see remark without proof in [7]). Assume that (ii) does not imply (i). As a consequence of remark (5) to Theorem 3.1 and from $a \geq b \geq c \geq d \geq e \geq f$ it follows: Since $(a, e, f)$ is a face-triple of $S_{\mathrm{sp}}$ we have $e+f \leq a$, which is equivalent to $2 e f \leq a^{2}-e^{2}-f^{2}$. Using this inequality and a specific representation of $\mathrm{D}\left(S_{\mathrm{sp}}\right)$ we get:

$$
\begin{aligned}
\mathrm{D}\left(S_{\mathrm{sp}}\right)= & 8 b^{2} e^{2} f^{2}-2 b^{2} \underbrace{\left(a^{2}-e^{2}-f^{2}\right)^{2}}_{\geq 4 e^{2} f^{2}}-2 e^{2} \underbrace{\left(f^{2}+b^{2}-d^{2}\right)^{2}}_{>0}-2 f^{2} \underbrace{\left(e^{2}+b^{2}-c^{2}\right)^{2}}_{>0} \\
& -2 \underbrace{\left(a^{2}-e^{2}-f^{2}\right)}_{>0} \underbrace{\left(f^{2}+b^{2}-d^{2}\right)}_{>0} \underbrace{\left(e^{2}+b^{2}-c^{2}\right)}_{>0} \\
< &
\end{aligned}
$$

which contradicts (ii). The result can be summarized as follows:

$S_{\mathrm{sp}}$ is tetrahedral $\Leftrightarrow \mathrm{D}\left(S_{\mathrm{sp}}\right)$ is positive.

\section{Anisometric tetrahedrons with given edge lengths}

How many (mutually) anisometric tetrahedrons having six given edge lengths can be formed, providing the spawning tetrahedron with these edge lengths exists?

First we consider the case of scalene tetrahedrons. The number of these tetrahedrons are found by constructive combinatorics as follows: Starting with the edge of largest length there are 5 possibilities for the length of the opposed edge. Any further edge can have the largest of the four remaining lengths, and, finally, there are 3! possibilities for the lengths of the last three edges. Hence, the number of anisometric scalene tetrahedrons is given by $5 \cdot 3 !=30$. The same result can be obtained by enumerative combinatorics: From the 6 ! possible sextuples always 4 ! determine (up to isometry) the same tetrahedron, and thus we have $6 ! / 4 !=30$ anisometric tetrahedrons. Note that, accordingly, the number of determinants $\mathrm{D}(S)$ in the "Outline of the proof" of Theorem 5.1 can be restricted to 30 . 
Which set $V$ consists of the smallest six successive integers such that all 30 anisometric scalene tetrahedrons with edge lengths from $V$ exist (see also remark in [7])? Since $n=7$ is the smallest positive solution of the diophantine inequality $\mathrm{D}\left(S_{\mathrm{sp}}\right)>0$ where $S_{\mathrm{sp}}=$ $(n+5, n+1, n+3, n+4, n+2, n)$ it follows from Theorem 5.1 and its remark (3):

$$
V=\{7,8,9,10,11,12\}
$$

Note the difference between the properties of the sets $U$ (4.1) and $V(6.1): U$ is completely tetrahedral, i.e., all tetrahedrons with edge lengths from $U$ exist, whereas $V$ guarantees the existence of all scalene tetrahedrons only.

We now turn to the general case. Let $p_{s}$ denote the number of anisometric tetrahedrons where $s$ is a sequence indicating how often every of the six given edge lengths occurs. The following list of all numbers $p_{s}$ can be verified by constructive combinatorics:

$$
\begin{aligned}
& p_{6}=1, p_{51}=1, p_{42}=2, p_{33}=3, p_{411}=2, p_{321}=4, p_{222}=6, \\
& p_{3111}=5, p_{2211}=9, p_{21111}=15, p_{111111}=30 \text { (scalene tetrahedrons). }
\end{aligned}
$$

For the case $p_{3111}=5$, the anisometric tetrahedrons are illustrated in Fig. 5, edge lengths being differentiated by line formats. Note that if the three solid lines represent the second largest edge length, then a tetrahedron as shown on the very left is a spawning one (independently of the order of the other three edge lengths). Taking any other order of the edge lengths a tetrahedron as shown on the very right would never be spawning.
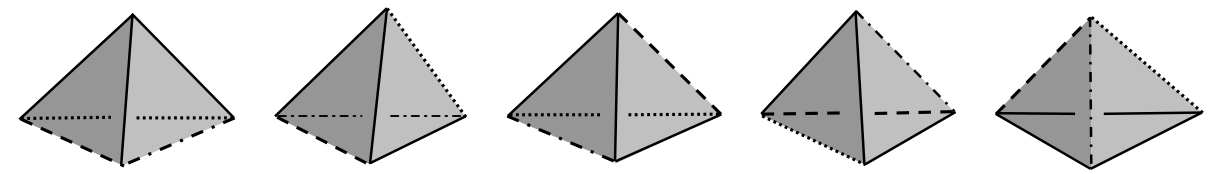

Fig. 5

Returning to the sets $U$ (4.1) and $V$ (6.1), we present two numbers of tetrahedrons: With the use of the above $p_{s}$ and simple enumerative combinatorics it is found that there are 2451 anisometric tetrahedrons with lengths from the completely tetrahedral set $U$ (this number was also derived with Polyas enumeration theory). With constructive combinatorics and the use of Theorem 3.1 to check tetrahedrality it is shown that there are 2360 anisometric tetrahedrons (scalene and non-scalene) with lengths from the set $V$; this number was derived with the help of a computer program.

\section{Tetrahedrality of golden and isosceles sextuples}

In this last section we deal with tetrahedrality of certain special sextuples. Two interesting examples are $S_{\mathrm{g}}=(a, a, b, a, b, b)$, named golden sextuple, and $S_{\mathrm{i}}=(a, b, c, a, b, c)$, named isosceles sextuple.

Theorem 7.1. A golden sextuple $S_{\mathrm{g}}=(a, a, b, a, b, b)$ is tetrahedral exactly if the ratio $r:=a / b$ lies in the interval $G=] 1 / \Phi, \Phi[$, where $\Phi$ is the golden ratio. 
Proof. We use Theorem 3.1: Calculation leads to

$$
\mathrm{D}\left(S_{\mathrm{g}}\right)=2\left(a^{2}+b^{2}\right)\left(a^{2}-a b-b^{2}\right)\left(b^{2}-a b-a^{2}\right) .
$$

Condition (ii) is satisfied exactly if $\left(r^{2}-r-1\right)\left(1-r-r^{2}\right)>0$, which is equivalent to $r \in G$. Since $r \in G$ implies $r \in$ ]0.5,2[ it follows that the face-triples $(a, a, b)$ and $(a, b, b)$ of $S_{\mathrm{g}}$ obey the L-inequality, meaning that condition (i) holds, too. (The reader will recall that, according to remark (5) to Theorem 3.1, it would be sufficient to consider only one face triple, which is trivial here because $a$ and $b$ are exchangeable.)

\section{Remarks}

(1) A golden tetrahedron, i.e., a tetrahedron determined by a golden sextuple, is given up to similarity by the ratio $r$ or by just one face angle. The relation between the base angles $\alpha$ and $\beta$ of two isosceles faces, which are anisometric in case $a \neq b$, is expressed by $4 \cos \alpha \cos \beta=1$ with $36^{\circ}<\alpha<72^{\circ}$ (see Fig. 6).

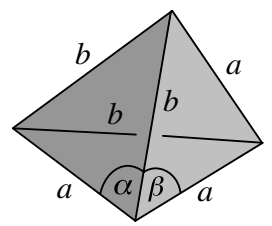

Fig. 6

(2) Any golden tetrahedron is spawning, so that, for $a \neq b$, the rearrangement of its edges always produces two additional anisometric tetrahedrons, according to $p_{33}=$ 3 (see Section 6).

Theorem 7.2. An isosceles sextuple $S_{\mathrm{i}}=(a, b, c, a, b, c)$ is tetrahedral exactly if it is acute-facial.

Proof. Starting with Theorem 3.1 one obtains by calculation

$$
\mathrm{D}\left(S_{\mathrm{i}}\right)=4\left(a^{2}+b^{2}-c^{2}\right)\left(a^{2}+c^{2}-b^{2}\right)\left(b^{2}+c^{2}-a^{2}\right) .
$$

Condition (ii) is satisfied exactly if the three bracket-factors are positive or two of them negative and one positive. The first case holds exactly if the four equal face-triples $(a, b, c)$ of $S_{\mathrm{i}}$ obey the Q-inequality. This means that $S_{\mathrm{i}}$ is acute-facial and, by Theorem 4.1, tetrahedral. The second case is impossible because at most one of the three factors can be negative (property of an unfulfilled triangle inequality).

\section{Remarks}

(1) That an isosceles tetrahedron, i.e., a tetrahedron determined by an isosceles sextuple, has only acute face angles may also be seen in a more direct way: Since the four vertex-triples are equal and since one vertex-triple is acute (Lemma 2.1), it follows that all twelve face angles are acute. Note that a planar figure is the net of an isosceles tetrahedron exactly if it consists of the four isometric triangles obtained by subdividing an acute triangle (see Fig. 7). 


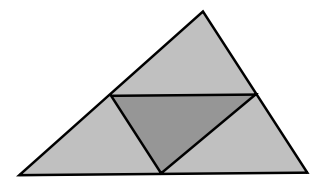

Fig. 7

(2) No isosceles tetrahedron with $a \neq b, a \neq c$ and $b \neq c$ is spawning.

\section{References}

[1] Blumenthal, L.M.: A note on the four-point property. Bull. Amer. Math. Soc. 39 (1933), 423-426.

[2] Blumenthal, L.M.: Some Imbedding Theorems and Characterization Problems of Distance Geometry. Bull. Amer. Math. Soc. 49 (1943), 321-338 (see p. 324).

[3] Blumenthal, L.M.: Theory and Applications of Distance Geometry. Oxford University Press, Oxford 1953 (see p. 100).

[4] Blumenthal, L.M.: A budget of curiosa metrica. Amer. Math. Monthly 66 (1959), 453-460 (see p. 455).

[5] Cayley, A.: On a theorem in the geometry of position. Cambridge Math. Journal 2 (1841), 267-271.

[6] Dekster, B.V.; Wilker, J.B.: Edge lengths guaranteed to form a simplex. Arch. Math. 49 (1987), 351-366.

[7] Herzog, F.: Completely tetrahedral sextuples. Amer. Math. Monthly 66 (1959), 460-464.

[8] Menger, K.: Untersuchungen über allgemeine Metrik. Math. Ann. 100 (1928), 75-163 (see pp. 133-136).

[9] URL: www.mathpages.com/home/kmath424.htm

Karl Wirth, André S. Dreiding

Organisch-Chemisches Institut

Universität Zürich

Winterthurerstrasse 190

CH-8057 Zürich, Switzerland

e-mail: wirthk@gmx.ch, asd@oci.unizh.ch 